\title{
DISPENSACIÓN DE MEDICAMENTOS EN LAS GRANDES FARMACIAS DE CHILE: ANÁLISIS ÉTICO SOBRE LA PROFESIÓN DEL QUÍMICO FARMACÉUTICO
}

\begin{abstract}
Adriana Marín Toro ${ }^{1}$
Resumen: Sobre la base de una investigación de carácter cualitativo, se describe el contexto de la dispensación de medicamentos en las grandes cadenas de farmacias de Chile, teniendo como protagonista a los profesionales químicos farmacéuticos quienes ocupan la función de directores técnicos de estas entidades. Se considera que el consumo y la economía están incrustados en todos los mecanismos de la vida, incluyendo la salud. Los medicamentos, en este sentido, son un claro ejemplo de un objeto de consumo utilizado cotidianamente por la población chilena, cuestión que hace necesaria una vigilancia política, social y ética al respecto. Se analizan las dimensiones sociales de los saberes técnicos de los químicos farmacéuticos, en base a los intereses de las farmacias en las que están contratados, de un éxito profesional medido en términos individuales y de la consecución de metas pecuniarias por sobre sanitarias, cuestión que incentiva una cultura que promueve el consumo de medicamentos, más que lo restringe. Estos profesionales son parte de un proyecto socioeconómico y sociopolítico particular capaz, incluso, de modificar las bases cognitivas de una profesión.
\end{abstract}

Palabras clave: dispensación de medicamentos, químicos farmacéuticos, dimensión social de los saberes técnicos, ética profesional

\section{Medicines dispensing in big pharmacies in Chile: ethical analysis about pharmaceutical chemist profession}

\begin{abstract}
Based on qualitative research, is described the context of dispensing drugs in "big pharmacy chains" in Chile, starring pharmaceutical chemists who have the role of technical directors of these entities. It is considered that the consumption and the economy are embedded in all the mechanisms of life, including health. Drugs in this sense, are a clear example of an everyday consumer object used by the chilean population, an issue which calls for a surveillance political, social and ethical about it. The social dimensions of the technical knowledge of pharmaceutical chemists are analyzed, based on the interests of pharmacies in which they are employed, a professional success measured in individual terms and achieving financial goals on health, an issue that encourages a culture that promotes the consumption of drugs, rather than restricting it. These professionals are part of a particular socio-economic and socio-political project capable even to modify the cognitive foundations of a profession.
\end{abstract}

Key words: dispensing of drugs, pharmaceutical chemists, social dimension of technical knowledge, professional ethics

Dispensaçáo de medicamentos em grandes farmácias no Chile: análise ética acerca da profissáo farmacêutica química

Resumo: Com base em uma pesquisa de caráter qualitativo, descreve-se o contexto da dispensação de medicamentos em grandes cadeias de farmácias no Chile, tendo como protagonista os profissionais farmacêuticos químicos que ocupam a função de diretores técnicos dessas entidades. São considerados o consumo e a economia como incorporados em todos os mecanismos da vida, incluindo a saúde. Os medicamentos, nesse sentido, são um claro exemplo de um objeto de consumo cotidiano pela populaçáo chilena, questáo esta, que exige a necessidade de um acompanhamento político, social e ético a este respeito. Analisou-se as dimensôes sociais do conhecimento técnico dos farmacêuticos químicos, com base nos interesses das farmácias onde eles estão contratados, de um êxito profissional, medido em termos individuais e do cumprimento de metas monetárias, sobre as de saúde, uma questão que incentiva uma cultura que promove o consumo de medicamentos, mais que os restringir. Estes profissionais fazem parte de um projeto socioeconômico e sociopolítico particular capaz, até mesmo, de alterar as bases cognitivas de uma profissão.

Palavras-chave: dispensação de medicamentos, farmacêuticos químicos, dimensão social do conhecimento técnico, ética profissional

\footnotetext{
${ }^{1}$ Facultad de Arquitectura y Urbanismo, Pontificia Universidad Católica de Valparaíso, Chile

Correspondencia: amarin@ead.cl
} 


\section{Introducción}

\section{Globalización: consecuencias globales y locales}

La economía está incrustada (embeddedness) en la $\operatorname{sociedad}(1)$, cuestión que define aspectos culturales, institucionales y temas económicos y no económicos. El concepto de "incrustación" es útil para explicar la imposibilidad de separar la economía de otras actividades en las sociedades moder$\operatorname{nas}(2)$.

En términos históricos, la segunda mitad del siglo XX es una época conocida como la "edad de oro del capitalismo" (3) y posee como característica preponderante la internacionalización de los mercados: en otras palabras, una reestructuración de la economía en la que el capital se valoriza en un número creciente de actividades y de lugares en el mundo. La interconexión, la movilidad del capital financiero a escala global y la profundización de mecanismos mercantiles, son elementos que forman parte de la definición del concepto de globalización $(4,5)$.

Una de las primeras industrias en romper fronteras y transformar social y culturalmente el mundo occidental luego de la Segunda Guerra Mundial es la perteneciente al campo de la química y la farmacología. Ejemplo característico es la introducción de los antibióticos, cuyos efectos sociales quedaron de manifiesto en los cambios demográficos que experimentaron los países del Tercer Mundo, debido a que atacaron eficazmente enfermedades infecciosas. Otros cambios culturales ocurrieron unas décadas más tarde con la revolución sexual de los años sesenta y setenta, por el uso de este fármaco y la píldora anticonceptiva(3).

El desarrollo de medicamentos es un elemento tecnológico importante en el campo de la salud(6), en específico para la prevención, el tratamiento, interrupción o modificación de enfermedades propias del ser humano. En este sentido, los fármacos simbolizan elementos de bienestar, salud y curación, pero estas ideas no responden a un orden natural o son ahistóricas, sino que nacen en un contexto social particular(7). Hoy, discutir sobre el mercado que envuelve a los medicamentos contiene una mirada escéptica, debido a las implicancias éticas de la mercantilización de nece- sidades humanas, posicionando al medicamento, social y culturalmente, como un objeto de consumo. Como consecuencia, se puede advertir prácticas cuestionables en los distintos actores sociales involucrados en el uso de medicamentos por parte de la población.

En Chile, por su parte, mientras en la década de los setenta cambia la base económica del país, también lo hace el área productiva nacional, en particular las organizaciones empresariales. Las nuevas formas de competencia estimulan la flexibilidad y la rapidez de estas instituciones, y ello se plasma en la descentralización y expansión territorial por medio de cadenas de empresas, nodos, asociaciones bajo la modalidad de franquicias, tratando de cubrir al máximo el mercado potencial. Ejemplos claros de este fenómeno en el país fueron los casos de: supermercados, tiendas por departamentos y farmacias (5).

\section{Presencia de las farmacias en Chile}

El mercado farmacéutico del país se caracteriza por grandes cadenas de farmacias, las que han logrado participar en la mayor parte de la distribución y comercialización de medicamentos y otros productos asociados, concentrando alrededor del 95\% del mercado(8). A 2012 estas farmacias obtuvieron ganancias de US\$1.500 millones, con un crecimiento anual, desde 2008 , que ha variado entre el 7 y el 8\%(8). Un 76,7\% de este total se explica por la venta de medicamentos que requieren receta médica y el 23,3\% restante corresponde a medicamentos de venta directa(8).

Estas grandes farmacias son instituciones en las que la mayoría de los químicos farmacéuticos ejercen labores como jefes técnicos de farmacia(9). A octubre del 2015 se contabilizaron en el país alrededor de 2.955 empresas de este tipo(10), farmacias que se pueden encontrar en los principales centros urbanos del país, permitiendo que los medicamentos sean un producto de fácil consumo para una parte importante de la población.

Por otra parte, la industrialización de los productos farmacéuticos tuvo un impacto global que afectó a la farmacia en todos sus aspectos, marcando el inicio del fin de la botica tradicional, debido a que los medicamentos ya no se podían crear con recur- 
sos propios, ni a un nivel individual, como antes lo hacían profesionales químicos farmacéuticos: "Y si podían hacerlo, no las podían igualar ni en costos ni en calidad de presentación. No podían tampoco copiarlas, porque estaban patentadas por sus inventores, quienes poseían recursos no sólo para defender sus derechos, sino para promoverlas y publicitarlas, generando una competencia nueva nunca antes vista. En este nuevo contexto económico, las farmacias fueron adquiriendo un rol más de distribuidores, que de productores" (9:153).

Las grandes farmacias son empresas que reflejan un juego de marketing con la salud, incluyen estrategias de negociaciones con laboratorios, cambios en el formato de la farmacia (tipo drugstore), donde no solo los medicamentos son los productos más importantes, también se puede econtrar juguetes, cosméticos y golosinas, además de alianzas con las principales casas comerciales del país para el pago con tarjetas de crédito, la retención de clientes mediante tarjetas de fidelización de clientes y acumulación de puntos para lograr distintos beneficios(11). Si los medicamentos fueran un bien de consumo como cualquier otro, estos datos no tendrían una mayor relevancia, pero los fármacos poseen una relación directa con las personas, pues implican un gasto importante de insumos médicos y otros dependen de ellos para conservar la salud y la vida.

En Chile, la relación entre mercado farmacéutico y sociedad se ha visto deteriorada en los últimos años, por haber traspasado límites éticos en su funcionamiento. Casos publicitados desde el año 2009 en adelante fueron las denominadas "canelas" (en jerga farmacéutica), incentivos económicos a los dependientes de las farmacias para que vendieran medicamentos que significaran una mayor renta utilitaria a la empresa; otro caso bullado en el país, durante el mismo año, fue la confirmación pública que realizó la Fiscalía Nacional Económica respecto a la colusión de precios en más de 200 medicamentos entre las grandes cadenas farmacéuticas.

El fraude comercial o las malas prácticas de mercado son los primeros antecedentes para generar un control social y político de los procesos de consumo, más allá de la compraventa en el intercambio de bienes(4). De modo similar a la relación entre sociedad y economía, el consumo hoy también está incrustado en múltiples mecanismos de la vida y, en ese sentido, se plantea como esencial la reflexión política, la participación de los actores sociales y la educación (formal e informal) respecto a qué es lo que se consume.

Si bien existe una estructura económica preponderante, no por ello los sujetos sociales están determinados a un estado de alienación y manipulación; puede ser que, por el contrario, representen a un ser soberano y libre en la persecución de intereses personales. En el "sujeto social" también conviven lógicas de confrontación, dominación, resistencia y cambio; en el caso de las prácticas de consumo, estas estructuran gran parte de nuestras identidades y las distintas formas de relaciones sociales en las que se produce y reproduce el poder, la dominación y la distinción(4).

Los cambios en la base económica del país, la organización productiva y la distribución territorial de las empresas, en particular en el caso de farmacias, implicaron giros profundos a nivel profesional en cuanto a objetivos y funciones. En el caso del mercado farmacéutico la principal repercusión la tuvieron los químicos farmacéuticos: "este prisma 'mercantilizado' de la farmacia, según la opinión de un número no menor de farmacéuticos, es - precisamente- lo que 'mató' a la farmacia tradicional y el ejercicio libre de la profesión. Incentivos como 'velocidad de rotación', 'premios por número de venta', 'ofertas de marcas propias', que enfatizan la venta en función de factores comerciales, por encima de objetivos sanitarios, son duramente criticados por desvirtuar la función cautelar de la farmacia en la dispensación. Más grave aún, que al desconocer el carácter esencial del medicamento, redujeron profesionalmente al farmacéutico a un rol meramente administrativo, marginándolo del mesón, que es un lugar donde sus conocimientos universitarios se facilitan para informar, orientar y aconsejar al enfermo sobre medicamentos"(9:140).

A luz de estos antecedentes, en Chile se comienza a tramitar una nueva "Ley de Fármacos", que finalmente se promulga en 2014 (Ley No 20.724), que "estableció normas relevantes para la regulación del mercado farmacéutico, definiendo un estatuto jurídico incipiente para el desarrollo de políticas 
públicas relacionadas con los medicamentos; sin embargo, varias de sus disposiciones quedaron sujetas en su aplicación a la dictación de reglamentos técnicos sobre la materia"(12:4).

La mencionada ley, entre otros elementos, prohíbe los incentivos a la venta de medicamentos, uno de los principales mecanismos de las grandes farmacias para la generación del sueldo variable de sus profesionales y de los auxiliares de farmacia. La normativa, debido a su reciente implementación, no permite observar sus actuales consecuencias en la relación entre las farmacias de cadena y sus dependientes. Sin embargo, estas instituciones tienen como uno de sus objetivos principales enfatizar aspectos comerciales por sobre los sanitarios, y es en este marco de acción que se analiza la profesión del químico farmacéutico.

La relación entre lo económico y lo social, sus repercusiones éticas, como también las diferentes escalas de análisis involucradas, es el trasfondo que persigue el presente artículo. Se quiere poner en discusión cómo la estructura económica también es capaz de estructurar otros ámbitos de la vida cotidiana, como el consumo, la salud e incluso profesiones, mediante discursos de químicos farmacéuticos empleados de las grandes cadenas de farmacias de Chile.

\section{Metodología}

La investigación tuvo un alcance exploratorio y descriptivo, y fue de carácter cualitativo. Se desarrolló en la Región de Valparaíso, importante macrozona de Chile que concentra la segunda mayoría de farmacias luego de la Región Metropolitana de Santiago(8). Se seleccionaron las farmacias privadas $y$, entre estas, las correspondientes a las grandes cadenas del sector farmacéutico, porque en estas organizaciones los químicos farmacéuticos, en su mayoría, realizan labores de directores técnicos.

La técnica de producción de datos fue la entrevista semiestructurada, momento en el que los entrevistados fueron expuestos a una situación concreta, en este caso su rol profesional en un contexto específico. Se centró la atención en la experiencia subjetiva de los participantes de esta investigación, basados en un guión de entrevista elaborado a partir del estudio previo del campo empírico y la teoría en la cual se enmarca el estudio, pero dejando el espacio para la no dirección de los entrevistados. Se realizó finalmente un total de 21 entrevistas. Los discursos fueron caracterizados y analizados como una red de palabras, acciones, símbolos, retóricas e intenciones. Discursos que no son sino reflejos de una voluntad que les precede; sociológicamente, toda práctica es producto de disposiciones previas, pero que se construyen en lo concreto, discursos que a su vez son multideterminados, conflictivos, contradictorios y transformadores(13-15). Los cuidados éticos, durante y después de realizada la investigación, estuvieron en el resguardo de la privacidad, la confidencialidad y el consentimiento de los entrevistados, quienes además dieron su aprobación para que las conversaciones fueran grabadas y luego reproducidas con fines académicos.

\section{Resultados}

\section{Quimicos farmacéuticos en las cadenas de farmacia}

"Es un deber administrar y hay que hacerlo bien para que el negocio sea rentable". Esta es una de las principales labores del químico farmacéutico al interior de las farmacias de cadena hoy en día. Apelar a un discurso técnico neutral, en este contexto, implica dejar fuera del análisis los aspectos éticos del ejercicio de la profesión, especialmente cuando existen incentivos a la venta y un éxito profesional medido en estos términos.

Las grandes farmacias son un centro de dispensación de medicamentos dirigido a un amplio público, cuya lógica es incentivar el consumo de medicamentos, más que restringirlo. En este lugar, el químico farmacéutico posee un conocimiento superior a la mayoría de los usuarios que compra medicamentos en estas empresas, por lo que el traspaso a fines poco claros y poco éticos, en un contexto de incentivo a la rentabilidad, se puede dar de manera cotidiana:

“...la parte administrativa es como cualquier negocio, como si vendiéramos zapatos, trabas, no sé, cualquier cosa, y la parte administrativa es un negocio que tiene productos que son rentables y otros que no lo son. Entonces hay que compatibilizar toda una parte que corresponde a medica- 
mentos, con la parte ética, con toda la parte comercial. O sea, si yo sé que dos medicamentos que tienen el mismo principio activo y uno me renta el triple, no me voy a quedar tranquila hasta vender el que me renta el triple" (QF, mujer, 50 años).

La distancia respecto del conocimiento sobre medicamentos deja a los consumidores finales en desventaja frente a un conocimiento técnico que les permita elegir, en estos términos, un medicamento por sobre otro. En consecuencia conceptos como bienestar, educación, salud y consumo(4) no pueden ser tratados como elementos separados, sino como centros de la planificación, participación, y toma de decisiones correspondientes a las políticas públicas, en este caso de salud y que aluden al consumo de medicamentos.

Actualmente, las decisiones en el proceso de dispensación de medicamentos en las grandes cadenas de farmacia están influenciadas por los intereses personales de los profesionales químicos farmacéuticos y los auxiliares de farmacias, en estrecha relación con los intereses promovidos por las instituciones que los contratan. Lo que existe hoy es una estructura económica y un arreglo de mecanismos institucionales que permiten y promueven el énfasis comercial, en este caso el medicamento como un bien de consumo, comercializado, facilitado al público y, en ocasiones, sin mayores restricciones de venta. En este contexto, el rol del químico farmacéutico es limitado, a sabiendas de las implicancias éticas que esto significa, pues su éxito profesional depende de la priorización de aspectos administrativos por sobre los sanitarios:

“...debiese haber un tema reglamentario que impidiera que la evaluación del desempeño del farmacéutico sea medida por temas comerciales y por temas de administración, cosa de dejar separados netamente la labor ética profesional del farmacéutico, de la labor de jefe de local de encargado como gerente de empresa, pero en estos momentos, así como está, te pueden medir por temas comerciales, los que podrían empañar las labores de salud pública, yo creo que gran parte de eso pasa por reconocer a la farmacia como una entidad preocupada de la salud pública" (QF, hombre, 38 años).

En las farmacias de cadena, el "buen farmacéutico" es aquel que "vende más", y es en este nivel donde es evaluado su desempeño; en un escenario ideal distinto, un "buen farmacéutico" sería quien restringe la venta de ciertos medicamentos o realiza acciones de farmacovigilancia a un número determinado de usuarios de la farmacia, cuestiones vinculadas a un uso racional de los medicamentos(16). Por el contrario, para cumplir las metas exigidas mes a mes, entran en juego una serie de disposiciones que limitan con la ética y la salud de la población, por la persecución de una favorable evaluación profesional. A pesar de las "buenas intenciones" para con el cliente, existe un compromiso con la mantención del empleo. En lo concreto, se pudo identificar una lealtad profesional divida en dos polos: en primer lugar con la institución que los contrata y, en segundo término, con la dimensión "ética de la técnica" (17); esto es, la práctica de los químicos farmacéuticos como una profesión relacionada al área de la salud:

1) Lealtad con la institución: "Bueno a nosotros nos evalúan, cumple o no cumple, pasó la meta o no pasó la meta, cumplió o no cumplió con los parámetros comerciales, y ahí va a haber una evaluación un poco rígida al respecto. La idea claramente para mí es cumplir; ahora, ser la "top" de las "top" no me interesa, porque en fondo es un lugar tan grande que tendría que comprometer a todos, y es súper difícil. Cuando tú manejas a cuatro personas es mucho más fácil comprometer a cuatro personas que comprometer a 18 vendedores en el mismo cuento. Entonces, de repente cuesta, a lo mejor no cumplimos todos los parámetros pedidos, pero sí siempre fue lo que ellos (la farmacia) nos pedían, ellos nos pedían un valor y de ahí pa'arriba, de ahí pa'arriba, esa es mi meta personal" (QF, mujer, 50 ańos).

2) Lealtad con la dimensión ética profesional: "Por ejemplo, este caballero está con una enfermedad cualquiera, pero con una loperamida se le pasa, pero claro, la loperamida vale CLP\$300 (USD\$ $0,45)$ CLP\$ 400 (USD\$ 0,55), entonces, ¿qué van a preferir? Darle un medicamento de marca, que te cuesta casi CLP\$6.000 (USD\$ 8,5), al final de cuentas puede que le haga el mismo efecto y no lo necesita, entonces ahí juega, juega con la ética sobre todo cuando tú ves que hay un cliente que no tiene para pagar CLP\$5.000 (USD\$7) o CLP\$6.000 (USD\$ 8,5), entonces jle vas a negar el otro más barato? No puedes, o sea, desde mi 
punto de vista no puedes negarlo, hay colegas que sí, que lo niegan y prefieren que el cliente se vaya antes de dar la opción del genérico" (QF, mujer, 29 años).

\section{Crisis sobre la identidad profesional de los químicos farmacéuticos en el mercado farmacéutico chileno}

Los químicos farmacéuticos, al definir quiénes son como profesionales, sobre la base de sus competencias técnicas y de la respuesta que ellos otorgan a las demandas sociales(18), destacaron tres roles:

1) profesionales de la salud (rol farmacéutico);

2) científicos (rol de químico)

3) y administrativos (rol de jefe técnico de farmacia).

La sociedad posee necesidades y aspiraciones en diferentes ámbitos, cuyo progreso técnico requiere de la aplicación de conocimientos especializados de alto nivel de complejidad, y estos son respondidos por los profesionales(18). En este caso, los químicos farmacéuticos están altamente especializados, con un nivel de conocimiento complejo en relación con los medicamentos; sin embargo, las necesidades a la que ellos responden en el contexto de las grandes cadenas de farmacias son de tipo administrativo, por sobre las de tipo sanitario o científico. Se acepta y se da continuo a un mito institucional(18), esto es, la legitimación de privilegios conquistados por las instituciones y que, al mismo tiempo, sirven de modelo que influye en la política de la institución y obliga a sus miembros a regirse por él. Dada sus competencias, los químicos farmacéuticos podrían tener un rol más relevante en la educación sobre el consumo de medicamentos de la población. No obstante, el incentivo económico sobre el mercado de los medicamentos en Chile es tan avasallador que es capaz de promover cambios en las bases de conocimiento de esta profesión:

“...quizás por lo mismo muchos colegas, entre los cuales me incluyo, porque tengo un magíster en administración, han querido ir perfeccionando esta área (administrativa) para trabajar de una forma más eficiente, justamente, para que te quede más tiempo disponible para hacer la labor que uno considera para la cual fuiste formado, que es estar ahí delante de la persona" (QF, hombre, 40 años).

“...nosotros (los químicos farmacéuticos) nunca hemos estado en la facultad de administración (en una universidad), porque si nuestro rol principal fuera administrar medicamentos, estaríamos en el área de administración, pero siempre se ha concebido a la profesión relacionada a la salud. Yo creo que la gente que entra a estudiar química y farmacia, entra creyendo que es una profesión de salud, entra creyendo que va a formar parte de un equipo, porque la carrera de farmacia siempre está asociada al área salud, y ¿por qué entonces le estamos dando la prioridad a la parte administrativa? ¡ah que los farmacéuticos somos ordenados! Claro y además la oficina es cómoda" (QF, mujer, 45 años).

En el ámbito descrito, lo que existe es un incentivo al consumo de medicamentos. Ideología que forma parte de un esquema habitual de la definición social de la realidad de este tipo de farmacias, legítimo para los propios profesionales, evidente en la dispensación de medicamentos y, a su vez, tradicionalmente respaldado por las políticas económicas del país. Por tanto, las decisiones profesionales basadas en conocimientos técnicos quedan en jaque por una serie de elementos de tipo social y económico, principalmente, que encausan la práctica cotidiana al cumplimiento de objetivos comerciales, en perjuicio de los de tipo sanitario.

"El rol de uno, de químico farmacéutico, de enseñar, por el tema económico, que también cumple la farmacia no permite - a mí no me permiteeducar a un paciente y decirle: 'sabe usted, mejor no compre esta marca, porque este antiflamatorio no va a tener los efectos colaterales que tiene la ergotamina'; pero por otro lado la empresa me dice usted tiene que cumplir y tiene que llegar a las metas y si no vende el producto que tiene tanta comisión, no puede llegar a las metas" (QF, hombre, 49 años).

Las farmacias de cadena, tipo drugstore, son lugares en los que se comercializan los medicamentos y además otras clases de artículos variados, como alimentos, cosméticos, juguetes, entre otros. El mensaje que se envía al consumidor es el siguiente: "Encontrar esta mezcla de productos en el expen- 
dio, es asumir que el medicamento se puede consumir tan fácil, autónoma e irresponsablemente como cualquier otro artículo. Obedece al mismo orden de cosas, las que se adquieren fácilmente y se consumen sin mayor cuidado, sin riesgos para su integridad física" (6). En este sentido, tanto la restricción a la venta de medicamentos como la liberación de la misma son soluciones de mercado y no resuelven necesariamente problemas sanitarios vinculados a aspectos culturales, simbólicos e ideológicos sobre el uso de medicamentos en Chile, en el que se promueve un consumo que en ocasiones es prescindible.

Dispensación de medicamentos como gestor de riesgos en las farmacias de cadena

El consumo, en tanto produce bienestar, también es gestor de riesgos(4). En las farmacias de cadena, en el proceso de dispensación de medicamentos, además de los químicos farmacéuticos participan los auxiliares de farmacias:

"Por el volumen de venta que hay en una farmacia, ahora, en la actualidad, fácilmente una farmacia mediana puede tener seis o siete vendedores auxiliares que en realidad son las manos del farmacéutico. Yo te digo, por ejemplo en las farmacias donde me ha tocado trabajar, las farmacias atienden alrededor de 1.000 personas diarias; entonces, para un farmacéutico, para un profesional, significa que tiene que delegar muchas de sus funciones técnicas a los auxiliares de farmacia" (QF, mujer, 30 años).

Los químicos farmacéuticos cumplen el rol de jefes y pueden capacitar a los auxiliares de farmacia sobre los productos disponibles, criterios y cuidados que deben tener con ciertos artículos. No obstante, la venta de medicamentos en estas empresas es de tipo comisionada, con un sueldo base mas bien bajo, que sube en la medida en que más productos son comercializados:

"Los vendedores tienen un sistema de contrato que en todas las cadenas, independiente a que digan que ya subió el sueldo base, siguen recibiendo ellos un sueldo que bordea el sueldo mínimo (CLP\$ 200.000 - USD\$280) y todo lo demás es su comisión, eso es. Ellos, lo que venden es lo que comen; entonces, ¿cómo yo puedo luchar contra eso?" (QF, hombre, 40 años).

Existen incentivos institucionales e individuales dirigidos a estos auxiliares para promover la venta indiscriminada de medicamentos, entre ellos hay productos que son más rentables que otros $y$, en consecuencia, la información que llega a los usuarios puede ser tendenciosa. La posición de los químicos farmacéuticos acerca de cómo se dispensan medicamentos en estas circunstancias son contradictorias, pues se sabe y asume que hay un incentivo a la venta de medicamentos pero, al mismo tiempo, ellos también forman parte de un sistema que les exige el cumplimiento de iguales objetivos. Paradójicamente, prácticas poco éticas de dispensación de medicamentos en las farmacias de cadena ayudan al éxito profesional de los químicos farmacéuticos como jefes técnicos de estas instituciones:

"Ahí cuesta conjugar la parte asistencial con la parte de la venta, porque también a ti te están evaluando de acuerdo con la venta del local, tienes una meta de venta todos los meses y tu sueldo depende de cumplir o no cumplir la meta” (QF, mujer, 35 años).

"yo tengo que hacer el juego comercial y pasa porque yo tengo que llegar a metas, yo tengo que vender, yo gano mientras yo más vendo, así de simple, y mi sueldo es un sueldo dependiente de las ventas, el sueldo de mis vendedores es dependiente de las ventas, porque si no tendría puro flojos ahí mirando el pasillo haciendo nada. Como buenos chilenos, uno necesita incentivos directos: mientras más vendes, más ganas, como si vendiera zapatos" (QF, mujer, 50 años).

Esta situación no es fácil de resolver, porque, al igual que un número importante de profesionales, los químicos farmacéuticos no son empresarios individuales, sino dependientes de organizaciones privadas y tienen importantes nexos de trabajo con ellos. En consecuencia, parte importante de los cambios que se pudiesen realizar en este sentido dependen de las restricciones y facilidades que tengan las entidades que los contratan, y hoy las farmacias mantienen un carácter comercial por sobre objetivos sanitarios. 


\section{Discusión}

\section{La función ética de las profesiones de salud}

La farmacia es un área de la salud que ha expandido su campo de acción en la última parte del siglo XX. Los farmacéuticos, asimismo, ya no solo preparan medicamentos, también brindan asesoramiento, información e instrucciones sobre su uso(19). No obstante, estos profesionales no pueden dispensar fármacos sin la autorización de un profesional legalmente facultado para prescribir, aunque sí pueden explicar sus efectos, además de recomendar medicamentos de venta libre. Es importante destacar que los químicos farmacéuticos son una importante fuente de información para el público en general, pues cumplen la función de reducir la distancia social con los elementos técnicos propios del medicamento(19).

La dispensación, en términos teóricos, implica más que la entrega de un producto. Sin embargo, en el contexto descrito, la función administrativa que cumplen los químicos farmacéuticos les impide desarrollar una labor educativa y de promoción de la salud. El objetivo de realzar a los profesionales de esta área se basa en que, en la sociedad contemporánea, las profesiones gozan de un prestigio social que las legitima como una voz importante en las definiciones de lo que se considera socialmente como "valores", concepciones de bien y de mal, aquello que parece justo e injusto, las aspiraciones razonables e irrazonables, lo normal, lo aceptable(20). En resumen, poseen influencia en las maneras de pensar y sentir de quienes se relacionan con ellos. Es una de las fuerzas creadoras de cultura e ideologías, tanto de aquellas que mantienen un cierto orden, como de las contestatarias, en vistas de promover una sociedad distinta(18).

En el caso de las profesiones asociadas con la salud, estas reflexiones poseen un componente aún mayor. Dada la secularización de la sociedad, la ética en la salud ha reemplazado ciertos mandatos morales: "la medicina no se limita solo a la prevención y tratamiento de las enfermedades: es ella quien define lo que constituye enfermedad y salud, y los límites entre estas dos condiciones, cosa que ya no es un problema netamente técnico sino un asunto de gran envergadura social" (18:30).
Las profesiones vinculadas al área de la salud son comprendidas como una colectividad que posee un conjunto de relaciones complejas, con derechos y obligaciones que los ligan con la sociedad. Esta definición corresponde a una concepción sociológica, que asocia la práctica profesional con una dimensión ética, más allá de los saberes técnicos, porque sus decisiones pueden llegar a condicionar la propia existencia cotidiana. Es por este motivo que las profesiones se pueden definir como una de las fuerzas creadoras de cultura, estatus que, en efecto, les otorga ciertas responsabilidades.

El concepto de "responsabilidad" en las profesiones implica la capacidad de responder de manera institucionalizada a necesidades sociales y, a su vez, le otorga un carácter público: "Da cuenta del grado en que la fe pública puede ser resguardada y es un elemento importante al calificar acciones, personas e instituciones relacionadas con el saber. Quiere decirse también que no basta apelar a las "infalibles" certidumbres del "mercado", que terminará "regulando" la oferta y la demanda de servicios en algún futuro incierto (...). Este imperativo se extiende especialmente a aquellos saberes llamados prácticos, que por eso mismo la tradición califica de "éticos" (17:29-30).

Se quiere destacar, además, que los proyectos socioeconómicos y sociopolíticos de un lugar no actúan independientes entre sí y las prácticas de los profesionales se posicionan al interior de estos ámbitos para ser llevadas a cabo. En este sentido, la estructura del mercado en el que los profesionales ofrecen sus servicios, las desigualdades sociales, la competencia del mercado, como la competencia por el poder social y político, son fenómenos en estrecho vínculo; cada uno de estos elementos condiciona a todos los demás y les otorgan límites y oportunidades a las profesiones(18).

Entonces, las prácticas y decisiones profesionales de los químicos farmacéuticos no actúan en un vacío. Es en el ajuste a su particular contexto donde se producen las contradicciones y tensiones empíricas observadas. Si bien se han dado a conocer traspasos éticos en la práctica de la dispensación de medicamentos en las grandes farmacias, en esta reflexión se quiso enfatizar a los profesionales participantes, porque ellos poseen un valor más que instrumental; $y$, como se ha expuesto, tienen la ca- 
pacidad de construir cultura, ya sea de aquella que promueve el cambio - en este caso, un uso racional de los medicamentos- o, por el contrario, que mantiene el statu quo, definido en el artículo como el incentivo al consumo de medicamentos en pos de intereses comerciales.

Si bien la Ley de Fármacos (No 20.724) intenta contrarrestar los efectos negativos de la concentración del mercado farmacéutico en ciertos agentes y el incentivo a la venta de medicamentos, es necesario hacer énfasis en la vigilancia política y social(4) antes planteada, y analizar cómo la medida toma forma en la realidad, qué intereses se privilegian, cuáles intervienen en la toma de decisiones profesionales, priorizando epistemológicamente las consecuencias prácticas, para realizar un análi- sis de la dimensión ética de este contexto(14). Las grandes farmacias tienen un propósito comercial. En estos términos, frente a posibles restricciones y modificaciones políticas lo más probable es que se generen nuevos arreglos institucionales que les permitan conseguir sus objetivos fundados en una racionalidad pecuniaria(21).

Finalmente, cabe destacar que el mercado farmacéutico en Chile, su funcionamiento e implicancias para los diferentes actores involucrados en él, es un campo de investigación que constantemente genera nuevos elementos para analizar sus dimensiones éticas, económicas y sociales. 
Dispensación de medicamentos en las grandes farmacias de Chile - Adriana Marín Toro

\section{Referencias}

1.

Polanyi K. The great transformation. The political and economic origins of our time. $1^{\text {a }}$ ed. Boston: Beacon; 1957.

2. Enguita M. Economía y sociología. Para un análisis sociológico de la realidad económica. Madrid: Siglo XXI de España Editores S.A.; 1998.

3. Hobsbawm E. Historia del Siglo XX. 3a. ed. Buenos Aires: Planeta; 2002.

4. Alonso L. Las políticas de consumo: transformaciones en el proceso de trabajo y fragmentación de los estilos de vida. Revista Española de Sociología 2004; 4: 7-50.

5. De Mattos C, Riffo L. Globalización, redes, nodos y dinámica metropolitana: El Gran Santiago en los noventa. En: De Mattos C, Figueroa O, Giménez R, Orellana A, Yáñez G. (eds.). Gobernanza y competitividad y redes: La gestión en las ciudades del siglo XXI. Santiago: Colección Rideal EURE-Libros; 2005: 31-63.

6. López J. Ética en la Industria Farmacéutica: entre la economía y la salud. Madrid: Eunsa; 2005.

7. Barajas C. Medicamentos y Símbolos. Revista Electrónica de Gastroenterología 2010 (Access: 2013 Junio 2). Available at: http://www.encolombia.com/medicina/gastroenterologia/gastro16201cronica.htm

8. Instituto de Salud Pública, Ministerio de Salud, Gobierno de Chile. Medicamentos en Chile: Revisión de la evidencia del mercado nacional de fármacos. Centro Nacional de Farmacoeconomía (CENAFAR) Subdepartamento de Estudios y ETESA Departamento de Asuntos Científicos Instituto de Salud Pública de Chile 2013. Diciembre. (Access: 2015 Noviembre 30). Available at: http://www.ispch.cl/sites/default/files/EstudioMedicamentos-22012014A.pdf

9. Guzmán E. Historia de la farmacia en Chile: 65 años. Santiago: Colegio Químico Farmacéutico y Bioquímico de Chile; 2008.

10. Instituto de Salud Pública, Ministerio de Salud, Gobierno de Chile. Farmacias Populares Octubre 2015. (Access: 2016 Febrero 22). Available at: http://www.recoleta.cl/wp-content/uploads/2015/10/ISP.pdf

11. Servicio Nacional del Consumidor. Grandes cadenas de farmacias: qué hay detrás de las promesas publicitarias 2006 Junio. (Access: 2009 Mayo 30). Available at: http://www.sernac.cl/estudios/detalle.php?id=1499

12. Ministerio de Salud, Gobierno de Chile. Regulación y acceso a medicamentos. Situación actual y proyecciones Enero 2016. Comisión Investigadora Cámara de Diputados. (Access: 2016 Febrero 22). Available at: http://web.minsal.cl/ministrade-salud-expone-sobre-acceso-a-medicamentos/

13. Alonso L. El Estructuralismo genético y los estilos de vida: consumo, distinción y capital simbólico en la obra de Pierre Bourdieu. Universidad de Navarra, Publicaciones y Recursos de Sociología y Áreas Afines (PURESOC) (s.f.). (Access: 2009 Mayo 5). Available at: http://www.unavarra.es/puresoc/pdfs/c_lecciones/LM-Alonso-consumo.PDF

14. Horwitz N. Dato y norma. El sentido de la investigación empírica en bioética. Santiago: Sociedad Chilena de Bioética; 2008.

15. Weber M. La ciencia como profesión. La politica como profesión. $3^{\mathrm{a}}$ ed. Madrid: Espasa Calpe; 2007.

16. Organización Mundial de la Salud. The Rational Use of Drugs. Report of the Conference of Experts (El Uso Racional de Medicamentos. Informe de la Conferencia de Expertos). Ginebra: OMS; 1985.

17. Lolas F. Bioética y Antropología Médica. Santiago: Mediterráneo; 2000.

18. Gyarmati M, Browne E, De la Barra A, Goic A, Gómez J, Guzmán M. Las Profesiones: dilemas de conocimiento y del poder. Santiago: Universidad Católica de Chile; 1984.

19. Cockerham W. Medical Sociology. 10a ed. New Jersey: Pearson/Prentice Hall; 2007.

20. Sapag-Hagar M. Ética, bioética, moral y ley: conceptos básicos. En Lolas F, ed. Ética y farmacia. Una perspectiva latinoamericana. $1^{\text {a }}$ ed. Santiago: CIEB; 2009: 25-44.

21. Busfield J. Pills, power, people: sociological understandings of the pharmaceutical industry. En: Brown P. (ed.). Perspectives in medical sociology. $4^{\mathrm{a}}$ ed. Illinois: Waveland Press, Inc.; 2008: 411-427.

Recibido: 22 de noviembre de 2015

Aceptado: 14 de febrero de 2016 